Supporting Information

\title{
Activating Basal Surface of Palladium by Electronic Modulation via Atomically Dispersed Nitrogen Doping for High-Efficiency Hydrogen Evolution Reaction
}

\section{Authors:}

Qianqian Yao ${ }^{1,2,3}$, Kai Yan ${ }^{4}$, Wenpeng Zhu ${ }^{1,2,3 *}$, Yue Zheng ${ }^{1,2,3}$

\begin{abstract}
Affiliations:
${ }^{1}$ School of Physics, Sun Yat-sen University, Guangzhou 510275, China

${ }^{2}$ State Key Laboratory of Optoelectronic Materials and Technologies, Sun Yat-sen University, Guangzhou 510275, China

${ }^{3}$ Centre for Physical Mechanics and Biophysics, School of Physics, Sun Yat-sen University, Guangzhou 510275, China

${ }^{4}$ Guangdong Provincial Key Laboratory of Environmental Pollution Control and Remediation Technology, School of Environmental Science and Engineering, Sun Yat-sen University, Guangzhou, 510006, China
\end{abstract}

*Email:zhuwp3@mail.sysu.edu.cn 


\section{Table of contents}

\section{Supplementary Figures}

Figure S1. Theoretical polarization curves of HER kinetics.

Figure S2. Charge transfer due to the N doping with the "N Atop" site occupied by hydrogen atom.

2. Note 1: theoretical polarization curves of HER kinetics

3. Note 2: partial atomic charges of the $\mathrm{N}$-doped Pd atoms and adsorbed hydrogen atom

\section{Methods}

DFT modeling, $\mathrm{N}$ doping energy and hydrogen adsorption free energy, exchange current density in volcano plot, theoretical polarization curve, charge density difference and partial atomic charge.

Supplementary References 


\section{Supplementary Figures}

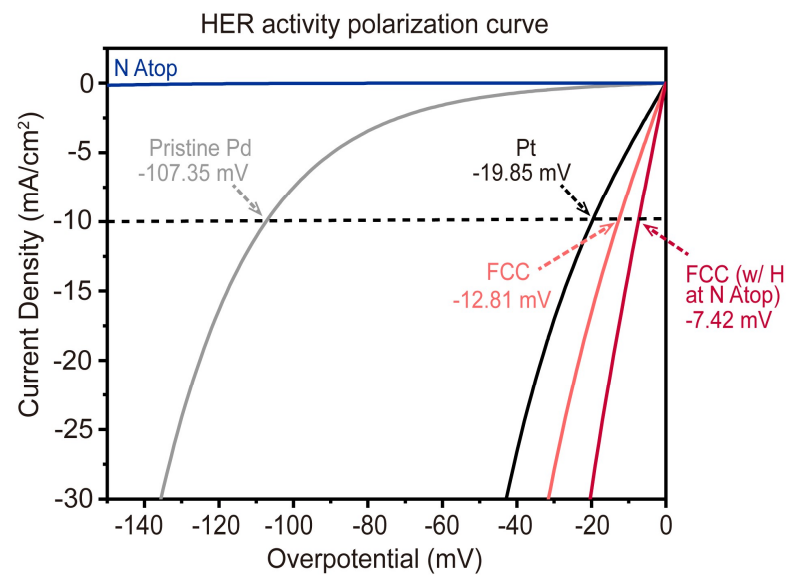

Figure S1. Theoretical polarization curves of HER kinetics. Calculated polarization curves of the current densities versus the applied overpotentials for three catalytic active sites on N-doped Pd surface ("N Atop": blue line; "FCC": pink line; "FCC (w/ H at N Atop)": red line), in comparison with the FCC sites on pristine Pd (grey line) surface and pristine Pt (black line) surface, with the transfer coefficient of 0.5 . The dashed line presents the reference of current density of $10 \mathrm{~mA} / \mathrm{cm}^{2}$. The dash arrows indicate the required overpotentials to achieve the current density of $10 \mathrm{~mA} / \mathrm{cm}^{2}$. 

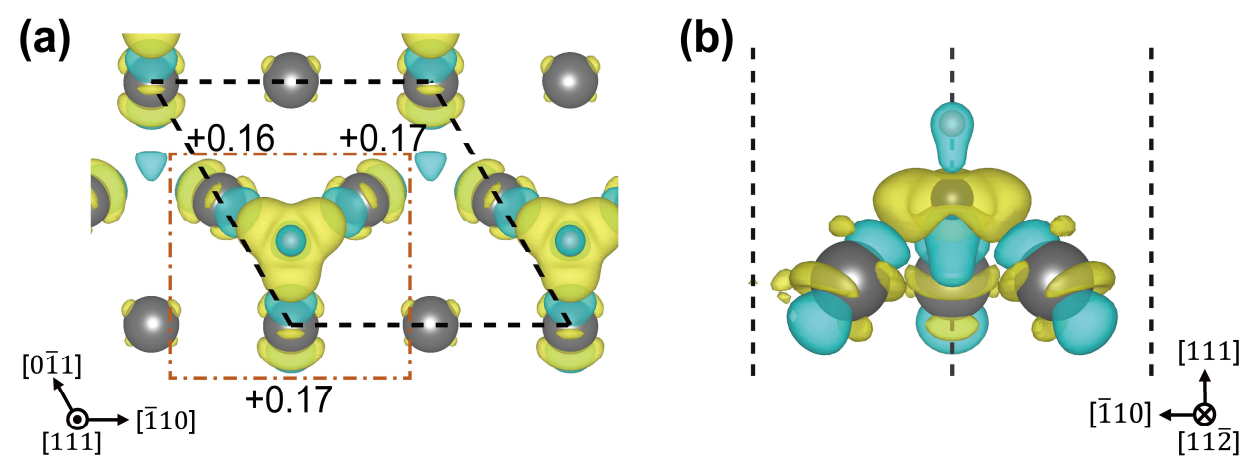

Figure S2. Charge transfer due to the N doping with the "N Atop" site occupied by hydrogen atom. $(\mathrm{a}, \mathrm{b})$ Charge density difference of Pd surface induced by the doped N atom with the hydrogen atom at "N Atop" site in the top (a) and side (b) views. The partial charges of Pd atoms bonded with the doped $\mathrm{N}$ atom are calculated and shown. The side view of (b) magnifies the region within the red dash-dot line in (a). The yellow and cyan iso-surfaces represent the electron accumulation and depletion with the values of \pm 0.008 e/Bohr ${ }^{3}$, respectively. 


\section{Note 1: theoretical polarization curves of HER kinetics}

To evaluate the HER kinetics of the basal surface of N-doped Pd and compare with the state-ofthe-art Pt electrocatalyst, we calculated the theoretical polarization curves of the current densities $(j)$ versus the applied overpotentials $(\eta)$ within the catalytic window as shown in Figure S1. Unlike the nonreactive "N Atop" site, the "FCC (w/ H at N Atop)" and "FCC" sites on the Ndoped Pd surface exhibit higher current density and lower overpotential than the FCC sites on pristine Pd and Pt surfaces. The descriptor, the required overpotential to achieve a current density of $10 \mathrm{~mA} / \mathrm{cm}^{2}$, has been commonly adopted for quantifying the HER kinetics. ${ }^{1,2}$ We compared these results of different catalytic active sites on the $\mathrm{N}$-doped $\mathrm{Pd}$, the pristine $\mathrm{Pd}$ and $\mathrm{Pt}$. The order of HER kinetics is "FCC (w/ H at N Atop)" site on N-doped Pd $(-7.42 \mathrm{mV})>$ "FCC" site on Ndoped $\mathrm{Pd}(-12.81 \mathrm{mV})>$ FCC site on pristine $\mathrm{Pt}(-19.85 \mathrm{mV})>$ FCC site on pristine $\mathrm{Pd}(-107.35$ $\mathrm{mV})>$ "N Atop" on $\mathrm{N}$-doped Pd. This trend proves that the atomically dispersed $\mathrm{N}$ doping, despite the sacrifice of "N Atop" sites, can substantially lower the overpotential and accelerate HER kinetics of the surrounding region, which hence changes the inert basal surface to be catalytically active for HER. 


\section{Note 2: partial atomic charges of the N-doped Pd atoms and adsorbed hydrogen atom}

To further understand the relation between the charge transfer induced by $\mathrm{N}$ doping and the hydrogen adsorption in HER, partial atomic charges were calculated for the N-doped Pd atoms and adsorbed hydrogen atom (Figure $4 \mathrm{c}$ and $4 \mathrm{~d}$ ). The N-bonded Pd atoms after hydrogen adsorption possess more positive values of partial charges $(+0.23 \mathrm{e}$ and $+0.22 \mathrm{e})$ than the Pd atoms $(-0.01$ e and -0.01 e) on the pristine surface, which is caused by the doped $\mathrm{N}$ atom dragging electrons away from the neighboring Pd atoms. Fewer electrons of these N-bonded Pd atoms can contribute to the binding with adsorbed hydrogen atoms, resulting in less negatively charged hydrogen atoms (-0.05 e) and the weakened hydrogen adsorption, which is in a great agreement with our previous energy calculations.

When the "N Atop" site is occupied at the "first step" in the hydrogen adsorption, the hydrogen atom anchored at the "N Atop" site can provide electrons for the doped $\mathrm{N}$ atom, and the N-bonded Pd atoms exhibit lower values $(+0.16 \mathrm{e},+0.17$ e and +0.17 e) of positive partial charges than the ones $(+0.20$ e,+0.19 e and +0.22 e) in the case of unoccupied N (Figure S2a and S2b). The anchored hydrogen atoms enable the N-bonded Pd atoms to withdraw electrons from the doped $\mathrm{N}$ atoms for re-strengthening the hydrogen adsorption in HER. This electron compensation increases the negative charge of the adsorbed hydrogen atom (-0.06 e) at "FCC" sites and decrease the positive charges of the N-bonded Pd atoms $(+0.20$ e and +0.19 e) as shown in Figure 4e. These results can explain the near-thermoneutral hydrogen adsorption and the superior HER activity at the "FCC" sites on the Pd basal surface after the top of the doped $\mathrm{N}$ atoms have been occupied. 


\section{Methods}

DFT modeling. The DFT modeling was performed by the Vienna ab initio simulation package $(\text { VASP })^{3}$ using a plane wave basis set in the projector-augmented-wave (PAW $)^{4}$ pseudopotentials. The Perdew-Burke-Ernzerhof (PBE) generalized gradient approximation to the exchangecorrelation functional is employed..$^{5}$ The plane-wave kinetic energy cutoff was fixed at $500 \mathrm{eV}$. The Pd (111) oriented surfaces were simulated by five-layer slab models built with a $2 \times 2$ supercell in a close-packed face-centered cubic (FCC) configuration, which were separated by a vacuum gap of $13 \AA$. The bottom three layers of the slabs were fixed, while the atoms in the top two layers were allowed to relax during the structure optimization. The Brillouin Zone was sampled by a Monkhorst-Pack k-point grid $^{6}$ of $4 \times 4 \times 1$. All the structures were considered as fully relaxed when the maximum force acting on each atom was less than $0.01 \mathrm{eV} \AA^{-1}$ and the energy change was less than $10^{-5} \mathrm{eV}$ in each electronic self-consistency loop. Non-self-consistent calculations with an energy convergence criterion of $10^{-5} \mathrm{eV}$ were also employed for the TDOS/PDOS. A denser Monkhorst-Pack k-point grid of $15 \times 15 \times 1$ was used for the TDOS/PDOS calculations.

$\mathbf{N}$ doping energy and hydrogen adsorption free energy. We considered four different $\mathrm{N}$ doping sites on the Pd surface, including FCC, hexagonal close packed (HCP), octahedral subsurface (OS) and tetrahedral subsurface (TS) sites. The FCC and HCP sites are located above the top layer with the projections resting in the center of the three neighboring Pd atoms. The doped $\mathrm{N}$ atom at the FCC site is staggered with the Pd atom at the sublayer forming the FCC stacking, or at the HCP site is aligned forming the HCP stacking. The OS and TS sites lie in the octahedral and tetrahedral 
interstices between the top layer and sublayer, which are directly below the FCC and HCP sites, respectively. The $\mathrm{N}$ doping energies $\left(\Delta E_{\mathrm{N}}\right)$ of each doping site were calculated as

$$
\Delta E_{\mathrm{N}}=E_{\mathrm{N}+\mathrm{slab}}-E_{\text {slab }}-0.5 \times E_{\mathrm{N}_{2}(\mathrm{~g})},
$$

where $E_{\mathrm{N}+\text { slab }}, E_{\text {slab }}$, and $E_{\mathrm{N}_{2}(\mathrm{~g})}$ represent the total energy of the Pd surface with doped $\mathrm{N}$ atom, the total energy of the pristine Pd surface, and the energy of a $\mathrm{N}_{2}$ molecule in gas phase, respectively.

We also considered four different hydrogen adsorption sites on N-doped Pd surface, including "N Atop", "HCP", "FCC" and "FCC (w/ H at N Atop)" sites. The "N Atop", "HCP”, "FCC" and "FCC (w/ H at N Atop)" represent the cases of hydrogen adsorptions on the top of the doped $\mathrm{N}$ atom, on the "HCP" site nearest to the doped $\mathrm{N}$ atom, on the "FCC" site nearest to the doped $\mathrm{N}$ atom without hydrogen on the top and on the "FCC" site nearest to the doped $\mathrm{N}$ atom occupied by a hydrogen on the top, respectively. Unlike the stable sites which can converge in DFT (like "N Atop", "FCC" and "FCC (w/ H at N Atop)"), the structure with hydrogen atoms binding on the "HCP" site is unstable and cannot converge, which suggests that it is unlikely for the HCP sites on the N-doped Pd surface to adsorb hydrogen atoms. The Gibbs free energies for hydrogen adsorption $\left(\Delta G_{\mathrm{H}}\right)$ of each stable site were obtained by

$$
\Delta G_{\mathrm{H}}=\Delta E_{\mathrm{H}}+\Delta E_{\mathrm{ZPE}}-T \Delta S
$$

where $\Delta E_{\mathrm{H}}$ is the differential hydrogen adsorption energy, $\Delta E_{\mathrm{ZPE}}$ is the difference in zero-point energy, $T$ is the ambient temperature as $298.15 \mathrm{~K}$, and $\Delta S$ corresponds to the entropy 
difference between adsorbed hydrogen atom and $\mathrm{H}_{2}$ molecule in the gas phase. The differential hydrogen adsorption energy $\Delta E_{\mathrm{H}}$ was derived as

$$
\Delta E_{\mathrm{H}}=\frac{1}{n}\left[E_{\mathrm{H}+\mathrm{slab}}-E_{\mathrm{slab}}-\frac{n}{2} E_{\mathrm{H}_{2}(\mathrm{~g})}\right]
$$

where $n$ is the number of $\mathrm{H}$ atoms in the calculation for considering the hydrogen coverage $(\theta) . n$ $=1$ corresponds to a hydrogen coverage of $\theta=1 / 4$ on the FCC sites of pristine Pd and Pt surface, and also corresponds to $\theta=1 / 3$ on the "FCC" and "FCC (w/ H at N Atop)" sites of N-doped Pd and $\theta=1$ on the "N Atop" sites of N-doped Pd, while $n=4$ corresponds to $\theta=1$ on the FCC sites of pristine Pd and Pt surface. The $E_{\mathrm{H}+\text { slab }}, E_{\text {slab }}$, and $E_{\mathrm{H}_{2}(\mathrm{~g})}$ represent the total energy of the Pd surface with adsorbed hydrogen atom, the total energy of the pristine Pd surface, and the energy of a $\mathrm{H}_{2}$ molecule in gas phase, respectively. The difference in zero-point energy $\Delta E_{\mathrm{ZPE}}$ can be calculated from the vibrational frequencies of the hydrogen atom between the adsorbed state and the gas phase. As the vibrational entropy of the hydrogen atom in the adsorbed state is negligible in comparison to half the vibrational entropy of the $\mathrm{H}_{2}$ molecule in the gas phase, ${ }^{7,8}$ the entropy difference $\Delta S$ can be given as

$$
\Delta S=-\frac{1}{2} S_{\mathrm{H}_{2}}^{0}=-65.34 \mathrm{~J} \cdot \mathrm{mol}^{-1} \cdot \mathrm{K}^{-1}=-0.6772 \mathrm{meV} \cdot \mathrm{K}^{-1},
$$

where $S_{\mathrm{H}_{2}}^{0}$ is the entropy of $\mathrm{H}_{2}$ in the gas phase at the standard condition. Different hydrogen coverages are considered for the theoretical calculations of hydrogen adsorption free energies. In this work, the Gibbs free energy for hydrogen adsorption on the FCC site of the pristine Pt surface is calculated as $-0.101 \mathrm{eV}(\theta=1 / 4)$ and $-0.017(\theta=1)$, which is in good agreement with previous study $(-0.09 \mathrm{eV}$ in $\theta=1 / 4$, and $-0.03 \mathrm{eV}$ in $\theta=1) .{ }^{7}$ To achieve the highest stability for HER (that 
is the smallest $\left.\left|\Delta G_{\mathrm{H}}\right|\right)$, the coverage of hydrogen adsorbed on the FCC sites of pristine Pd and pristine Pt, and the "N Atop" sites of N-doped Pd were chosen as 1, on the "FCC" and "FCC (w/ $\mathrm{H}$ at N Atop)" sites of N-doped Pd were chosen as $1 / 3$.

Exchange current density in volcano plot. The theoretical exchange current density $\left(i_{0}\right)$ in the volcano plot of this work was calculated based on the Gibbs free energy $\left(\Delta G_{\mathrm{H}}\right.$ ) for hydrogen adsorption, which follows the simple mean-field microkinetic model developed by Nørskov and coworkers under full hydrogen coverage and the benchmarked active site density of $\mathrm{Pt}^{7}$ and assuming transfer coefficient $(\alpha)$ of $0.5 .^{7,9,10}$ The theoretical exchange current density ${ }^{1}$ for the exothermic hydrogen adsorption $\left(\Delta G_{\mathrm{H}}<0\right)$ forms the left leg of the volcano and can be expressed by

$$
i_{0}=-e k_{0}\left(\frac{N_{\mathrm{a}}}{A}\right) \frac{1}{1+\exp \left(-\alpha \cdot \Delta G_{\mathrm{H}} / k_{\mathrm{B}} T\right)}
$$

where $e$ is the elementary charge, $k_{0}$ is the rate constant with a value of $200 \mathrm{~s}^{-1}$ per site fitted to give a reasonable overall magnitude of the rate, $N_{\mathrm{a}} / A$ is the number of active sites $\left(N_{\mathrm{a}}\right)$ per area $(A), k_{\mathrm{B}}$ is the Boltzmann constant, and $T$ is the ambient temperature and set to be 298.15 K. For the endothermic hydrogen adsorption $\left(\Delta G_{\mathrm{H}}>0\right)$, the theoretical exchange current density forms the right leg of the volcano and is given as

$$
i_{0}=-e k_{0}\left(\frac{N_{\mathrm{a}}}{A}\right) \frac{1}{1+\exp \left(-\alpha \cdot \Delta G_{\mathrm{H}} / k_{\mathrm{B}} T\right)} \exp \left(-\alpha \cdot \Delta G_{\mathrm{H}} / k_{\mathrm{B}} T\right)
$$


Hydrogen coverages and active site densities for different cases are considered for the theoretical calculations of exchange current density. ${ }^{11-13}$ We benchmark the active site density $\frac{N_{\mathrm{a}}}{\mathrm{A}}$ of $\mathrm{Pd}$ surface to that in Pt using $1 \times 10^{15}$ sites $/ \mathrm{cm}^{2}{ }^{2}$ The active site densities for the hydrogen adsorption on the "N Atop", "FCC" and "FCC (w/ H at N Atop)" sites are modified by $\frac{N_{\mathrm{N} \text { Atop }}}{A}=\frac{1}{4}\left(\frac{N_{\mathrm{Pt}}}{A}\right)$, $\frac{N_{\mathrm{FCC}}}{A}=\frac{3}{4}\left(\frac{N_{\mathrm{Pt}}}{A}\right)$ and $\frac{N_{\mathrm{FCC}(\mathrm{w} / \mathrm{H} \text { at N Atop })}}{A}=\frac{3}{4}\left(\frac{N_{\mathrm{Pt}}}{A}\right)$, respectively.

Theoretical polarization curve. The HER polarization curve of the current density $(j)$ versus the applied overpotential $(\eta)$ is calculated using the well-known Butler-Volmer equation ${ }^{1}$ as

$$
j=i_{0}\left(e^{\frac{(1-\alpha) n F \eta}{R T}}-e^{\frac{-\alpha n F \eta}{R T}}\right)
$$

where $\alpha$ is the transfer coefficient, which assumed to be $0.5,{ }^{7,9} n$ is the number of electrons involved in the reaction ( $n=2$ in the hydrogen evolution reaction ${ }^{14}$ ), $F$ and $R$ are the Faraday and gas constants, $T$ is the ambient temperature and set to be $298.15 \mathrm{~K}$. $i_{0}$ is the theoretical exchange current density in the volcano plot calculated based on the hydrogen binding free energy $\left(\Delta G_{\mathrm{H}}\right)$.

Charge density difference and partial atomic charge. The spatial charge density difference $\left(\Delta \rho_{\mathrm{N}+\mathrm{Pd}}\right.$ ) for the $\mathrm{N}$ doping on Pd surface in Figure $4 \mathrm{a}$ and $4 \mathrm{~b}$ is visualized by

$$
\Delta \rho_{\mathrm{N}+\mathrm{Pd}}=\rho_{\mathrm{N}+\mathrm{Pd}}-\rho_{\mathrm{Pd}}-\rho_{\mathrm{N}},
$$


where $\rho_{\mathrm{N}+\mathrm{Pd}}$ and $\rho_{\mathrm{Pd}}$ are the space charge densities of the Pd surface with and without $\mathrm{N}$ doping, respectively. $\rho_{\mathrm{N}}$ is the electron charge density of a single $\mathrm{N}$ atom.

The charge density difference $\left(\Delta \rho_{\mathrm{H}+\mathrm{Pd}}\right)$ for the hydrogen adsorption on Pd surface in Figure $4 c-d$ is given by

$$
\Delta \rho_{\mathrm{H}+\mathrm{Pd}}=\rho_{\mathrm{H}+\mathrm{Pd}}-\rho_{\mathrm{Pd}}-\rho_{\mathrm{H}}
$$

where $\rho_{\mathrm{H}+\mathrm{Pd}}$ and $\rho_{\mathrm{Pd}}$ are the space charge densities of the Pd surface with and without hydrogen adsorption, respectively. $\rho_{\mathrm{H}}$ is the electron charge density of a single hydrogen atom.

The charge density difference for the effect of the doped $\mathrm{N}$ atom with the "N Atop" site occupied by a hydrogen atom as shown in Figure S2 is calculated by the spatial charge density difference $\Delta \rho_{(\mathrm{Nw} / \mathrm{H})+\mathrm{Pd}}$ as

$$
\Delta \rho_{(\mathrm{Nw} / \mathrm{H})+\mathrm{Pd}}=\rho_{(\mathrm{N} \mathrm{w} / \mathrm{H})+\mathrm{Pd}}-\rho_{\mathrm{Pd}}-\rho_{(\mathrm{N} \mathrm{w} / \mathrm{H})}
$$

where $\rho_{(\mathrm{N} w / \mathrm{H})+\mathrm{Pd}}$ is the space charge densities of the N-doped Pd surface with the occupied "N Atop" site, $\rho_{\mathrm{Pd}}$ is the space charge densities of the pristine Pd surface, and $\rho_{(\mathrm{N} \mathrm{w} / \mathrm{H})}$ is the electron charge density of $\mathrm{N}$ and $\mathrm{H}$ atoms at the same positions of the modelling cell.

The partial charges for the doped $\mathrm{N}$ atom, the adsorbed hydrogen atoms and $\mathrm{Pd}$ atoms were calculated by the Bader charge analysis ${ }^{15}$ to quantitatively evaluate the degree of charge transfer. 


\section{Supplementary References}

1. Ekspong, J.; Gracia-Espino, E. Theoretical analysis of surface active sites in defective $2 \mathrm{H}$ and $1 \mathrm{~T}^{\prime} \mathrm{MoS}_{2}$ polymorphs for hydrogen evolution reaction: quantifying the total activity of point defects. Adv. Theory Simul. 2020, 3, 1900213.

2. Er, D.; Ye, H.; Frey, N. C.; Kumar, H.; Lou, J.; Shenoy, V. B. Prediction of enhanced catalytic activity for hydrogen evolution reaction in Janus transition metal dichalcogenides. Nano Lett. 2018, 18, 3943-3949.

3. Kresse, G.; Furthmüller, J. Efficient iterative schemes for $a b$ initio total-energy calculations using a plane-wave basis set. Phys. Rev. B 1996, 54, 11169-11186.

4. Blöchl P E. Projector augmented-wave method. Phys. Rev. B 1994, 50, 17953-17979.

5. Perdew, J. P.; Burke, K.; Ernzerhof, M. Generalized gradient approximation made simple. Phys. Rev. Lett. 1996, 77, 3865-3868.

6. Monkhorst, H. J.; Pack, J. D. Special points for Brillouin-zone integrations. Phys. Rev. B 1976, 13, 5188-5192.

7. Nørskov, J. K.; Bligaard, T.; Logadottir, A.; Kitchin, J. R.; Chen, J. G.; Pandelov, S.; Stimming, U. Trends in the exchange current for hydrogen evolution. J. Electrochem. Soc. 2005, 152, 23-26.

8. Chase Jr, M. W. NIST-JANAF Thermochemical Tables. J. Phys. Chem. Ref. Data, Monogr. 1998, 9 .

9. Greeley, J.; Jaramillo, T. F.; Bonde, J.; Chorkendorff, I.; Nørskov, J. K. Computational highthroughput screening of electrocatalytic materials for hydrogen evolution. Nat. Mater. 2006, 5, 909-913.

10. Hansen, H. A.; Varley, J. B.; Peterson, A. A.; Nørskov, J. K. Understanding trends in the electrocatalytic activity of metals and enzymes for $\mathrm{CO}_{2}$ reduction to CO. J. Phys. Chem. Lett. 2013, 4, 388-392.

11. Che, M. Nobel Prize in chemistry 1912 to Sabatier: organic chemistry or catalysis? Catal. today 2013, 218, 162-171.

12. Medford, A. J.; Vojvodic, A.; Hummelshøj, J. S.; Voss, J.; Abild-Pedersen, F.; Studt, F.; Bligaard, T.; Nilsson, A.; Nørskov, J. K. From the Sabatier principle to a predictive theory of transition-metal heterogeneous catalysis. J. Catal. 2015, 328, 36-42. 
13. Seh, Z. W.; Kibsgaard, J.; Dickens, C. F.; Chorkendorff, I. B.; Nørskov, J. K.; Jaramillo, T. F. Combining theory and experiment in electrocatalysis: insights into materials design. Science 2017, 355, eaad4998.

14. Shinagawa, T.; Garcia-Esparza, A. T.; Takanabe, K. Insight on Tafel slopes from a microkinetic analysis of aqueous electrocatalysis for energy conversion. Sci. Rep. 2015, 5, 13801.

15. Tang, W.; Sanville, E.; Henkelman, G. A grid-based Bader analysis algorithm without lattice bias. J. Phys. Condens. Matter 2009, 21, 084204. 